\section{A CROSS-SECTIONAL STUDY TO EVALUATE THE ETIOLOGICAL PROFILE OF HEMOPTYSIS AND IT'S CORRELATION WITH SEVERITY}

KEY WORDS: Hemoptysis, Etiology, Severity, bronchoscopy, chest CT

\section{Dharamendra Kumar Gupta*}

\section{R.K Jenaw}

Resident Doctor, Department of Respiratory Medicine, SMS Medical College, Jaipur. *Corresponding Author

Senior Professor, Department of Respiratory Medicine, SMS Medical College, Jaipur.

Introduction:- Hemoptysis is the spitting of blood that originated in the lungs or bronchial tubes. The patient's history should help determine the amount of blood and a focused physical examination can lead to the diagnosis in most cases. Chest radiographs often aid in diagnosis and assist in using two complementary diagnostic procedures, fiberoptic bronchoscopy and high-resolution computed tomography, which are useful in difficult cases and when malignancy is suspected.

Materials and Methods: -We prospectively evaluated 100 patients with hemoptysis, aged $\geq 18$ years, presented in our hospital during l-year study period. After applying inclusion and exclusion criteria, study population was selected. Patient underwent thorough clinical, general and respiratory examination. Initial routine blood, sputum and radiological evaluation was done in form of chest radiographs. Further chest computed tomography (CT) and bronchoscopic evaluation was done to find the etiology in cases diagnosis was not confirmed in chest radiograph. Final diagnosis was made, and association is seen between various demographic characteristics, comorbidities, severity of hemoptysis, radiological findings and diagnostic yield of different modalities was made.

Results:- Tuberculosis was found to be the most common etiology (39\%), followed by bronchogenic carcinoma(14\%) and bronchiectasis (13\%). The majority of the cases had mild (48\%) and moderate (39\%) hemoptysis with a small proportion of patients (13\%) with severe hemoptysis. The diagnostic yield of CT Chest was $73 \%$, that of FOB was $69.5 \%$ and the combined yield using both the modalities was calculated to be $82.6 \%$.

Conclusion:- Pulmonary tuberculosis remains a leading cause of hemoptysis because of high prevalence but still it is an alarming symptom and require thorough workup to find out the exact etiology. The combined use of bronchoscopy and chest CT probably gives the best diagnostic yield.

\section{INTRODUCTION:-}

Hemoptysis is a common and important symptom in clinical practice and accounts for $10-15 \%$ of all pulmonary consultations ${ }^{1}$. Hemoptysis is defined as the spitting of bloodderived from the lungs or bronchial tubes as a result of pulmonary or bronchial haemorrhage ${ }^{2}$. Hemoptysis is classified as non-massive or massive based on the volume of blood loss; however, there are no uniform definitions for these categories $^{1}$. In most cases, a hemoptysis is a self-limiting event but in fewer than $5 \%$ it may be severe or massive, representing a life-threatening condition that warrants urgent investigations and treatment $t^{3,4,5}$.The symptoms are distressing for the patient and frequently raise a concern about serious underlying disorders, including lung cancer ${ }^{6}$. Therefore, a radical evaluation of a patient with even a little amount of hemoptysis is vital. Haemoptysis is an alarming symptom, and the management depends upon the aetiology.

While precise epidemiologic data are lacking, infection is the most common cause of hemoptysis, accounting for 60 to 70 percent of cases ${ }^{7}$. But since there is an extensive differential diagnosis for hemoptysis, a step-wise approach to the evaluation of this symptom is appropriate. The mortality rate from untreated massive hemoptysis is more than $50 \%$. Therefore, prompt recognition of severe hemoptysis and identification of its causes is essential to initiate an adequate treatment and to avoid fatal complications ${ }^{8}$.

Chest X-ray, thorax computed tomography (CT), and fiberoptic bronchoscopic examination (FOB) are the most frequently used diagnostic techniques but there is no consensus about the procedure of choice in certain conditions.

So, this study prospectively evaluates the clinico-radiological and pathological profile of patients presenting with hemoptysis in our hospital and to determine the relation of etiology to the severity and find out the strategies used for localization of site of bleeding.

\section{MATERIALS AND METHODS: -}

A tertiary hospital-based, descriptive type of observational study was conducted at the Department of Respiratory Medicine, SMS Medical college, Jaipur, during the year 20192020 with hemoptysis aged 18 years. The patients with known coagulopathies, bleeding originating from the nasal cavities (i.e., epistaxis) or the gastrointestinal tract (i.e., hematemesis) and extra pulmonary causes of hemoptysis were excluded from our study. Demographic data were entered in a predesigned proforma. Routine blood investigations were carried out. Thorough general and respiratory system examination was done. Patients were classified according to severity. Mild - <30ml/24hr, Moderate 30-100ml/24hr, Severe $100-500 \mathrm{ml} / 24 \mathrm{hr}$, Massive - $\geq 500 \mathrm{ml}$ within 24 hours or where consequent hemodynamic instability occurs.

Diagnosis of pulmonary tuberculosis was based on chest radiography and sputum examination for acid-fast bacilli. For bronchogenic carcinoma, the diagnosis was based on histopathology. Bronchitis was diagnosed when a patient had symptoms consistent with an upper airway infection and a normal chest radiograph.

Diagnosis of bronchiectasis was confirmed by highresolution $\mathrm{CT}$ of thorax. Final diagnosis was established by consensus after consideration of all clinical, radiographic, HRCT-chest, and bronchoscopic findings. Patients for whom etiology could not be established were diagnosed as hemoptysis of unknown etiology. Conservative management was the preferred mode of treatment. All patients were followed-up for one year. The statistical analysis has been carried out using SPSS statistical package (version 9). The descriptive statistics mean and standard deviation (SD) were used to describe the finding. Paired t-test was used to test the difference in mean between two dependent groups. Fisher exact test was used to test the difference in two dependent variables. P-value $\leq 0.05$ was taken as the level of significance.

RESULTS: -In our study, $98 \%$ of patients were diagnosed at 
the end of the study, the remaining $2 \%$ of patients remained undiagnosed. The study population comprised $72 \%$ males and $28 \%$ females with a sex ratio of $2.5: 1$. In patients diagnosed with tuberculosis, males were 2 times of females. The maximum population belonged to the age group $>50$ years. All cases of malignancy were above the age of 50 years. The mean age of males was 49.04 years and that of females was 37.07 years. The smoking population comprised mainly of males $(68.06 \%)$. The main comorbid conditions observed in the study population were hypertension and diabetes.

The microbiological correlation for sputum culture positivity with hemoptysis patients is $32.8 \%$. Most of patients (82\%) presented with cough followed by fever $(46 \%)$ while all cases of malignancy presented with chest pain. The most common etiology was found to be tuberculosis, both active and inactive $(39 \%)$, bronchogenic carcinoma was the second most common cause for hemoptysis accounting for $14 \%$ of the total, followed by bronchiectasis (13\%). The majority of the cases had mild (48\%) and moderate (39\%) hemoptysis with a small proportion of patients $(13 \%)$ with severe hemoptysis.

Chest CT was performed for 83 cases, of which pulmonary lesions were detected in 76 cases $(91.57 \%)$. The diagnostic yield of CT Chest was $73 \%$, that of FOB was $69.5 \%$ and the combined yield using both the modalities was calculated to be $82.6 \%$. CT scan thorax yield was $79 \%$ in our study with a major diagnosis like bronchiectasis and tuberculosis. We had a yield of $65 \%$ in FOB which included tuberculosis, pneumonia, and bronchogenic carcinoma.

\begin{tabular}{|c|c|c|c|}
\hline \multicolumn{5}{|c|}{ Table 1. Characteristics of patients admitted with } \\
hemoptysis \\
\hline Age Group(years) & Male (n=28) & Female (n=72) & Total \\
\hline $18-30$ & $10(35.71 \%)$ & $10(13.89 \%)$ & $20(20 \%)$ \\
\hline $31-50$ & $14(50 \%)$ & $24(33.33 \%)$ & $38(38 \%)$ \\
\hline$>50$ & $4(14.29 \%)$ & $38(52.78 \%)$ & $42(42 \%)$ \\
\hline Mean Age & 49.04 years & 37.07 years & \\
\hline Smoking Status & & & \\
\hline Smokers & $4(14.29 \%)$ & $49(68.06 \%)$ & $53(53 \%)$ \\
\hline Non-smokers & $24(85.71 \%)$ & $23(31.94 \%)$ & $47(47 \%)$ \\
\hline Diabetes status & & & \\
\hline Yes & - & - & $22(22 \%)$ \\
\hline No & & & $78(78 \%)$ \\
\hline $\begin{array}{c}\text { Associated } \\
\text { symptoms }\end{array}$ & & & \\
\hline Cough & - & - & $82 \%$ \\
\hline Fever & - & - & $46 \%$ \\
\hline Chest pain & - & - & $30 \%$ \\
\hline Dyspnoea & - & - & $26 \%$ \\
\hline
\end{tabular}

Table 2. Radiological findings of study subject

\begin{tabular}{|c|c|c|}
\hline Radiological findings & Frequency & Percentage \\
\hline \multicolumn{3}{|c|}{ Based on Chest X-Ray (Zones involved) } \\
\hline More than 2 zones & 23 & $23.00 \%$ \\
\hline RUZ & 24 & $24.00 \%$ \\
\hline LUZ & 9 & $9.00 \%$ \\
\hline RLZ & 5 & $5.00 \%$ \\
\hline LLZ & 7 & $7.00 \%$ \\
\hline RMZ & 8 & $8.00 \%$ \\
\hline LMZ & 5 & $5.00 \%$ \\
\hline Normal & 19 & $19.00 \%$ \\
\hline Total & 100 & $100.00 \%$ \\
\hline \multicolumn{3}{|c|}{ Based on Chest CT results } \\
\hline Thorax CT results & Frequency & Percentage \\
\hline Mass lesion & 12 & $14.46 \%$ \\
\hline Aneurysm & 2 & $2.41 \%$ \\
\hline Tree in Bud appearance & 1 & $1.20 \%$ \\
\hline Bronchiectasis & 14 & $16.87 \%$ \\
\hline Consolidation & 14 & $16.87 \%$ \\
\hline Cavity & 18 & $21.69 \%$ \\
\hline
\end{tabular}

\begin{tabular}{|c|c|c|}
\hline Cyst & 1 & $1.20 \%$ \\
\hline Fibrosis & 4 & $4.82 \%$ \\
\hline Fungal Ball & 8 & $9.64 \%$ \\
\hline Broncho vascular distortion & 3 & $3.61 \%$ \\
\hline Nodules & 1 & $1.20 \%$ \\
\hline Normal & 5 & $6.02 \%$ \\
\hline Total & 83 & $100.00 \%$ \\
\hline
\end{tabular}

Table 3. Distribution of various etiologies associated with hemoptysis and their association with severity.

\begin{tabular}{|c|c|c|c|c|c|c|}
\hline Etiology & $\begin{array}{l}\text { Mild } \\
(n=48)\end{array}$ & $\begin{array}{c}\text { Moderat } \\
e \\
(n=39)\end{array}$ & $\begin{array}{l}\text { Severe } \\
(n=13)\end{array}$ & Total & $\begin{array}{c}\mathbf{P} \\
\text { value }\end{array}$ & \begin{tabular}{|c|} 
Test \\
perfor \\
med
\end{tabular} \\
\hline $\begin{array}{c}\text { Active } \\
\text { TB }\end{array}$ & $\begin{array}{c}11 \\
(22.92 \%)\end{array}$ & $\begin{array}{c}13 \\
(33.33 \%)\end{array}$ & \begin{tabular}{|c|}
4 \\
$(30.77 \%)$
\end{tabular} & \begin{tabular}{|c|}
28 \\
$(28 \%)$
\end{tabular} & \multirow[t]{9}{*}{0.791} & \multirow{9}{*}{\begin{tabular}{|c|} 
Fisher \\
Exact \\
test
\end{tabular}} \\
\hline Old TB & $\begin{array}{c}8 \\
(16.67 \%)\end{array}$ & \begin{tabular}{|c|}
2 \\
$(5.13 \%)$
\end{tabular} & \begin{tabular}{|c|}
1 \\
$(7.69 \%)$
\end{tabular} & $\begin{array}{c}11 \\
(11 \%)\end{array}$ & & \\
\hline $\begin{array}{c}\text { Aspergil } \\
\text { loma }\end{array}$ & $\begin{array}{c}6 \\
(12.50 \%)\end{array}$ & \begin{tabular}{|c|}
5 \\
$(12.82 \%)$
\end{tabular} & \begin{tabular}{|c|}
2 \\
$(15.38 \%)$
\end{tabular} & $\begin{array}{c}13 \\
(13 \%)\end{array}$ & & \\
\hline $\begin{array}{c}\text { Bronchie } \\
\text { ctasis }\end{array}$ & $\begin{array}{c}6 \\
(12.50 \%)\end{array}$ & $\begin{array}{c}5 \\
(12.82 \%)\end{array}$ & \begin{tabular}{|c|}
2 \\
$(15.38 \%)$
\end{tabular} & $\begin{array}{c}13 \\
(13 \%)\end{array}$ & & \\
\hline $\begin{array}{c}\text { Lung } \\
\text { carcino } \\
\text { ma }\end{array}$ & $\begin{array}{c}8 \\
(16.67 \%)\end{array}$ & $\begin{array}{c}6 \\
(15.38 \%)\end{array}$ & $\begin{array}{c}0 \\
(0 \%)\end{array}$ & $\begin{array}{c}14 \\
(14 \%)\end{array}$ & & \\
\hline $\begin{array}{c}\text { Pneumo } \\
\text { nia }\end{array}$ & \begin{tabular}{|c|}
2 \\
$(4.17 \%)$ \\
\end{tabular} & $\begin{array}{c}2 \\
(5.13 \%) \\
\end{array}$ & \begin{tabular}{|c|}
1 \\
$(7.69 \%)$ \\
\end{tabular} & $\begin{array}{c}5 \\
(5 \%)\end{array}$ & & \\
\hline $\begin{array}{c}\text { Cryptog } \\
\text { enic }\end{array}$ & $\begin{array}{c}2 \\
(4.17 \%) \\
\end{array}$ & $\begin{array}{c}0 \\
(0 \%)\end{array}$ & $\begin{array}{c}0 \\
(0 \%)\end{array}$ & $\begin{array}{c}2 \\
(2 \%)\end{array}$ & & \\
\hline Others & $\begin{array}{c}5 \\
(10.42 \%)\end{array}$ & $\begin{array}{c}6 \\
(15.38 \%)\end{array}$ & $\begin{array}{c}3 \\
(23.08 \%)\end{array}$ & $\begin{array}{c}14 \\
(14 \%)\end{array}$ & & \\
\hline Total & $\begin{array}{c}48 \\
(100 \%)\end{array}$ & $\begin{array}{c}39 \\
(100 \%)\end{array}$ & $\begin{array}{c}13 \\
(100 \%)\end{array}$ & $\begin{array}{c}100 \\
(100 \%)\end{array}$ & & \\
\hline
\end{tabular}

\section{DISCUSSION: -}

A total of 100 patients were included in the study. The study population was divided into three age groups i.e. 18-30,31-50, $>50$ years. The mean age of males was 49.04 years and that of females was 37.07 years as shown in Table 1. In our study, tuberculosis constituted the maximum proportion of cases in all age groups. This was also found in the study by Weaver et al. ${ }^{9}, \mathrm{~F}$ Soares Pires et al. ${ }^{10}$, in their study reported that 28 out of 78 patients who were diagnosed with malignancy were above the age of 40 years. This coincides with our findings of $100 \%$ of patients with lung carcinoma above age 50 years. Higher age is a risk factor for malignancy and hence older patients presenting with hemoptysis should be investigated appropriately so that we may detect malignancy at an early stage and improve survival rates.

The male-to-female ratio in our study was found to be 2.5:1. Males have a higher incidence of tuberculosis as well as malignancy, as they are more exposed to tubercle bacilli due to workplaces and more smoking prevalence in males ${ }^{11,12}$. This is validated by the study of Shouyong $\operatorname{Tan}^{13}$ and Bharade et al. ${ }^{14}$. Bronchiectasis was more commonly diagnosed in females. Similar results were found in study by Maria Tsoumakidou et al. ${ }^{15}$

The different associated symptoms at presentation included cough (82\%), fever (46\%), chest pain (30\%) and dyspnoea $(26 \%)$. Out of this, cough was the most important symptom followed by fever as shown in table 1. These findings correlated with the study of M Thirumaran et al ${ }^{16}$, where cough was present in $71 \%$ cases. Chest pain was found in $100 \%$ patients diagnosed with malignancy with P-value of less than 0.001 i.e., statistically significant. This suggests that chest pain with hemoptysis is an important symptom of malignancy.

The most common comorbidities associated with hemoptysis in our study was found to be hypertension and diabetes. These could be causative for hemoptysis as well as attributable to underlying diseases like COPD and other 
vascular disorders. Thus, associated clinical features and comorbidities are supportive in reaching a final diagnosis.

Microbiological correlation for sputum culture positivity with haemoptysis patients is $(32.8 \%)$. Various organisms were found in Culture \& Sensitivity, in which Klebsiella (7.8\%), Pseudomonas (7.8\%) and Enterococci (4.68\%) accounted for the most common organisms.

Radiological findings association shows more than two zone involved radiological lesion attributed (23\%), RUZ (24\%) and LUZ ( $9 \%$ ) among most common zones as shown in table 2 . In our study, $91 \%$ patients of hemoptysis had radiological positive finding on initial investigation of Chest X-ray. Fiden et $\mathrm{a}^{17}$, validate these results. Normal chest radiograph was also found in 19 study subjects. In our study chest CT was performed for 83 cases, out of which pulmonary lesions were detected in 76 cases $(91.57 \%)$. CT scan is superior to chest radiograph in ability to identify both the anatomic origin and underlying cause of hemorrhage. The manifestation of active tuberculosis on CT Chest included cavitatory changes in 8 cases, consolidation in 6 cases, tree in bud nodules in 1 case and miliary nodules in 1 case. In a study by Yeon et al. ${ }^{18}$, finding of cavity on CT Chest was a consistent finding with the diagnosis of tuberculosis. Tree in bud signs also correlate with the activity of the disease. ${ }^{19}$. Bronchiectasis was noted in CT scan of 14 patients (16.87\%). Aspergilloma was seen in 8 cases $(9.64 \%)$. Lung mass was seen in 12 cases (14.46\%). In 5 patients there was no obvious radiological abnormality noted \{Table 2\}. Fiden et $\mathrm{al}^{17}$, Joseph et $\mathrm{al}^{20}$ also found similar findings. Thus, patients even with non-specific findings on CT Chest must be further evaluated as they may have a serious underlying disorder and the important diagnosis.

As evident from our study, both active and inactive tuberculosis is an important cause of hemoptysis (39\%). The study by AT Abal et $\mathrm{al}^{21}$ which was done on 52 patients had similar results. They also reported the most common cause to be tuberculosis in $32.7 \%$ of cases followed by bronchiectasis $(21.2 \%)$. Another study by F. Soares Pires et $\mathrm{al}^{22}$ also revealed tuberculosis as the commonest cause of hemoptysis in $32.2 \%$ followed by bronchiectasis and malignancy in $15 \%$ each. However, in our study, bronchogenic carcinoma was the second most common cause for hemoptysis accounting for $14 \%$ of the total, followed by bronchiectasis (13\%). Other Indian studies have not reported such a finding, probably because of poor diagnostic facilities and the cases were, therefore, included in the 'undiagnosed' category.

Medical reviews from different parts of the world revealed that the etiological pattern of hemoptysis has changed in the developed countries but in our country, however, the pattern remained the same, tuberculosis is still the leading cause of it as is evident from the present study. Bronchiectasis was present in $13 \%$ in our study which is almost similar to $13.6 \%$ reported by Rao in $1960^{23}$.

Our study population comprised of $48 \%$ of mild, $39 \%$ of moderate and $13 \%$ of severe hemoptysis. It was found that majority of patients with tuberculosis, pneumonia and malignancy presented with mild and moderate hemoptysis. Tuberculosis with bronchiectasis and aspergilloma were the major causes of severe hemoptysis $(69.3 \%)$. There have been similar results in other study done by Hirschberg et al. ${ }^{3}$. In the present study, the diagnostic yield of CT Chest was $73 \%$, that of FOB was $69.5 \%$ and the combined yield using both the modalities was calculated to be $82.6 \%$. This is in concordance with various studies like that of $F$ Sores Pires et $a .^{10}$ and Hirschberg et al. ${ }^{3}$ where the combined yield was $76.7 \%$ and $67 \%$ respectively. Chest CT has been shown to be accurate in the diagnosis of a wide range of bronchial abnormalities. Its complementary use with bronchoscopy gives a greater positive yield of pathology and is useful for excluding malignancy in high-risk patients. Patients with recurrent or unexplained hemoptysis may need additional laboratory evaluation to establish a diagnosis. Bronchoscopy is a reliable method in locating the site of bleeding ${ }^{1}$. It also allows collection of histologic samples but is not useful in detecting peripheral tumours. The combined use of bronchoscopy and CT chest has the best yield in evaluating haemoptysis.

Despite the use of CT Chest and FOB, 2\% cases remained undiagnosed at the end of our study. In previous studies, these cases accounted for $7-25 \%$ of hemoptysis cases and the clinical course was usually minor and benign ${ }^{24}$.

\section{Limitations: -}

The major limitation of the study is the choice of the study site, as Institute of Respiratory Diseases being a tertiary referral centre, hence the sample cannot be representative of the general population.

\section{CONCLUSION:-}

Pulmonary tuberculosis remains a leading cause of hemoptysis because of high prevalence of tuberculosis in our country but lung malignancies especially in male smokers is at rising trend. Although majority of patients presented with mild to moderate hemoptysis but still it is an alarming symptom and require thorough workup to find out the exact etiology.

\section{REFERENCES: -}

1. Santiago S, Tobias J, Williams AJ. A reappraisal of the causes of haemoptysis. Arch Intern Med 1991; 151:2449-2451.

2. Bam N, Das SK, Lamsal M. Clinico-radiological and pathological profile of patients of hemoptysis admitted in a tertiary care centre in Nepal. JIOM;2017.39:1:29-32.

3. Hirshberg B, Biran I, Glazer M, Mordechai R. Haemoptysis: aetiology, evaluation and outcome in a tertiary referal Hospital. Chest 1997; 112: 440444.

4. Webb WR, Gamsu G, Glazer G. Computerised tomography of the abnormal pulmonary hilum.J Comput Assist Tomography 1981;5:485-490.

5. Ofori A, Steinmetz AR, Akaasi J, Asafu Adjaye Frimpong GA, Norman BR, Obeng-Baah J, et al. Pulmonary aspergilloma: An evasive disease. Int J Mycobacteriol 2016;5:235-9.

6. Hamilton W, Peters TJ, Round A, Sharp D. What are the clinical features of lung cancer before the diagnosis is made-A population-based case control study? Thorax 2005;60:1059-65.

7. Harrison TR, Braunwald E. Hemoptysis. In: Harrison's Principles of internal medicine. 15th ed.NewYork:McGraw-Hill, 2001:203-6.

8. Domoua K, N'Dhatz M, Coulibaly G, et al. Hemoptysis: Main etiologies observed in a pneumology department in Africa. Rev Pneumol Clin 1994;50: 59-62.

9. Nawal SK, Heda MR. Hemoptysis: A prospective analysis of 110 cases. Asian J Biomed Pharm Sci 2013;3:1-3.

10. Sores Pires F, Teixeira N, Ceelho F, Damas C. Hemoptysis - etiology, evaluation, and treatment in a university hospital. Rev Port Pneumol 2011;17(1):7-14.

11. Kolappan C, Gopi P. Tobacco smoking and pulmonary tuberculosis. Thorax 2002;57:964-6.

12. Bates MN, Khalakdina A, Pai M, Chang L, Lessa F, Smith KR. Risk of tuberculosis from exposure to tobacco smoke: A systemic review and meta analysis. Archives of internal medicine 2007;167:335-42.

13. Tan S, Sun D, Zhang T, Li Y. Risk factors for hemoptysis in Pulmonary Tuberculosis Patients from southern china: A retrospective study. JTR Dec 2014;9:173-80.

14. Bharade R, Mhaisekar D, Jadhav S. Clinical profile of primary lung cancer and role of bronchoscopy. 2015;34:5187-93

15. Tsoumakidou M, Chrysofakis G, Tsiligiannim I, Maltezakis G, Siafakas NM Tzanakis N. Prospective analysis of 184 hemoptysis cases - diagnostic impact of chest X-Ray, computed tomography, bronchoscopy. Respiration 2006;73:808-14

16. Thirumaran M, Sundar R, Sutcliffe IM, Currie DC. Is investigation of patients with haemoptysis and normal chest radiograph justified? Thorax 2009; 64:854.

17. Fidan A, Ozdogan S, Oruc O, Salepci B, Ocal Z and Caglayan B. Hemoptysis: a retrospective analysis of 108 cases. Respiratory Medicine. September 2002; $96,(9) ; 677-80$

18. Jeong YJ and Lee KS. Pulmonary tuberculosis: up-to-date imaging and management. Journal of Roentgenology 2008;191:834-44.

19. Lee KS, Im JG. CT in adults with tuberculosis of the chest: characteristic findings and role in management. AJR 1995;164:1361-7.

20. Joseph T, Nair S, James PT. Clinical-Radiological, Pathological Profile and Treatment Outcome of Patients with Haemoptysis. J PulmRespir Med 2017;7:437.

21. Abal AT,Nair PC and cherian J. Hemoptysis: etiology, evaluation and outcome a prospective study in a third-world country. Respiratory Medicine. 2001; 95, 548-52.

22. Sores Pires F, Teixeira N, Ceelho F, Damas C. Hemoptysis - etiology, evaluation, and treatment in a university hospital. Rev Port Pneumol 2011;17(1):7-14

23. Rao PU. Hemoptysis as a symptom in a chest clinic. Ind J Chest Diseases.1960; 2:219.

24. Herth F, Ernst $A$, Becker HD. Long term outcome and lung cancer incidence in patients with hemoptysis of unknown origin. Chest 2001;120:1592-4. 